\title{
Variabilidade genética do peso ao nascer e seleção para crescimento em bubalinos do Estado do Pará, Brasil
}

\author{
Juliana Flor de AGUIAR ${ }^{1 *}$, Cintia Righetti MARCONDES², José Ribamar Felipe MARQUES 3 , \\ Pedro Alejandro VOZZI ${ }^{4}$, Raimundo Nonato Colares CAMARGO JÚNIOR ${ }^{5}$, Larissa Coelho MARQUES ${ }^{6}$, \\ Ronyere Olegário ARAÚJO7, Ricardo José GUNSKI ${ }^{8}$

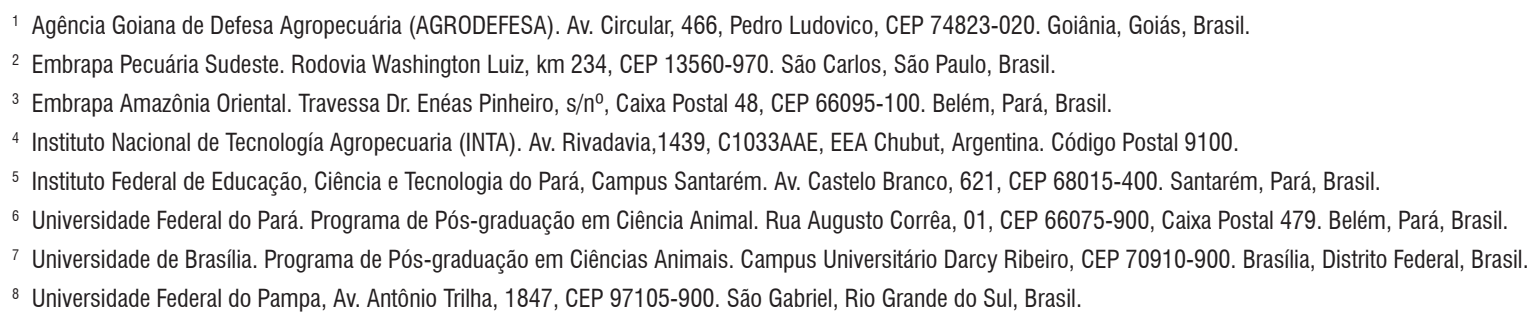

\section{RESUMO}

$\mathrm{O}$ peso ao nascer constitui característica produtiva de elevada importância zootécnica, devido à sua relação com a taxa de sobrevivência à desmama e com os pesos nas demais fases de desenvolvimento do animal, quer seja para a produção de carne, leite ou em animais que se destinam à reprodução. O objetivo deste trabalho foi obter estimativas de herdabilidade e tendências fenotípicas e genéticas do peso ao nascer, em bubalinos do Estado do Pará, Brasil. Foram calculadas as estatísticas descritivas e realizado o teste de Normalidade de Shapiro-Wilk por meio do pacote estatístico Statistical Analisys System. As estimativas de herdabilidade foram obtidas por inferência Bayesiana. $\mathrm{O}$ peso ao nascer apresentou média de 36,6 kg. O modelo de análise considerou como fixos os efeitos de sexo, ano de nascimento e composiçáo racial do animal e como efeitos aleatórios animal, efeito materno e residual. A distribuição da herdabilidade direta apresentou-se platicúrtica (achatada) e com maior assimetria, tendo uma distribuição bimodal com a primeira moda próxima a 0,10 e a segunda próxima a 0,30; a materna apresentou-se trimodal, com picos bem próximos a 0,15 e outro menos evidente próximo a 0,20 . A tendência genética direta do peso ao nascer mostrou-se negativa $\left(-0,03 \mathrm{~kg} \mathrm{ano}^{-1}\right)$ e a tendência genética materna próxima à zero $\left(0,001 \mathrm{~kg}\right.$ ano $\left.{ }^{-1}\right)$, ainda que a tendência fenotípica tenha sido positiva $\left(0,156 \mathrm{~kg} \mathrm{ano}^{-1}\right)$. Existe variabilidade genética possível de ser trabalhada em um programa de melhoramento, no entanto, pouco foi feito quanto à seleção para crescimento em búfalos no Estado do Pará.

PALAVRAS-CHAVE: Herdabilidade, inferência Bayesiana, tendência.

\section{Genetic variability of birth weight and selection for growth of water buffaloes from State of Pará, Brazil}

\section{ABSTRACT}

Birth weight is a performance parameter of great zootechnical importance for both meat and dairy production, as well as for breeding animals, mainly due to its relation to survival rate at weaning, and the weight of animals throughout their developmental and growth phases. Therefore, the objective of this work was to establish heritability estimates, as well as phenotypic and genetic trends of birth weight of water buffaloes from State of Pará, Brazil. Descriptive statistics were calculated and the Shapiro-Wilk Normality test was performed with a Statistical Analysis System package. Heritability estimates were established by Bayesian inference. BW was $36.6 \mathrm{~kg}$ in average. The statistical model considered sex, year of birth and breed composition of the animals as fixed effects, and animal, maternal and residual as random effects. Direct heritability was platykurtic (flattened) and with higher asymmetry, presenting a bimodal distribution with the first mode close to 0.10 , and the second mode close to 0.30 ; the maternal heritability was trimodal, with peaks very close to 0.15 , and another, less evident, close to 0.20 . The direct genetic trend of birth weight was negative $\left(-0.03 \mathrm{~kg} \mathrm{year}^{-1}\right)$ and maternal genetic trend was close to zero $\left(0.001 \mathrm{~kg}\right.$ ano $\left.{ }^{-1}\right)$, even though the phenotypic trend had been positive $\left(0.156 \mathrm{~kg} \mathrm{year}^{-1}\right)$. There is genetic variability to be addressed in a breeding program, however, very little was done as far as selection for growth of water buffaloes in the State of Pará.

KEYWORDS: Bayesian inference, trends, heritability. 


\section{INTRODUÇÃO}

Para os bubalinos, assim como nas demais espécies animais, o peso ao nascer constitui característica produtiva de elevada importância zootécnica, devido à relação existente entre o peso ao nascimento e a taxa de sobrevivência à desmama como, também, sua relação com os pesos e ganhos de peso nas demais fases de desenvolvimento do animal, quer seja para a produção de carne, leite ou para animais que se destinam à reprodução (Lopes et al. 2008).

De acordo com Nogueira et al. (1997), bezerros bubalinos que apresentam maiores pesos ao nascimento tendem a ganhar peso mais rapidamente, antes e depois da desmama, tornando importante a detecção de fatores genéticos e de meio que influenciam o peso dos búfalos ao nascer.

O peso ao nascer é também uma das primeiras expressóes fenotípicas do genótipo individual e pode auxiliar na seleção precoce de animais em programas de melhoramento genético (Lopes et al. 2008). A variabilidade observada nesta característica possui origem genética e não genética o que, segundo Pereira (2008), torna necessária a quantificação do que seria de natureza genética ou ambiental. Nesse sentido, conhecer os valores de herdabilidade é fundamental para definição do esquema de seleção mais adequado.

Portanto, o presente estudo tem como objetivo avaliar as estimativas de herdabilidade e de tendências fenotípica e genética do peso ao nascer de búfalos das raças Murrah, Mediterrâneo e mestiços provenientes de dois rebanhos comerciais e da Embrapa Amazônia Oriental, localizados no Estado do Pará, Brasil.

\section{MATERIAL E MÉTODOS}

$\mathrm{O}$ arquivo de dados utilizado no estudo continha informaçóes do animal, pai, mãe (nome e registro), sexo, data de nascimento, composição racial (com sete níveis entre Mediterrâneo x Murrah: 3/4 Murrah, 7/8 Murrah, 15/16 Murrah, 25/32 Murrah e 31/32 Murrah), fazenda (com quatro níveis) e peso ao nascer. Foram utilizados 566 registros de peso ao nascer de progênies de 16 pais e 269 mães distintos, nascidos entre fevereiro de 1981 e junho de 2009 em quatro fazendas do Estado do Pará, Brasil, localizadas nos municípios de Belém (Embrapa), Ipixuna (comercial), Santarém (Embrapa) e Santo Antônio do Tauá (comercial) com média de 2,1 progênies/búfala. Os animais nasceram em regime exclusivo de pastagens, predominando as pastagens naturais no período de menor disponibilidade de chuvas. Os pesos eram aferidos com no máximo $48 \mathrm{~h}$ pós-parto, dependendo do manejo adotado em cada fazenda e de acordo com a época do ano.

Foram realizadas estatísticas descritivas e teste de Normalidade de Shapiro-Wilk (procedimento CAPABILITY) utilizando o pacote estatístico Statistical Analysis System (SAS 2002). Para a obtenção dos componentes de variância foram utilizados programas de análise em linguagem Fortran (Misztál 2001), sendo formatado arquivo de parâmetros para o modelo estudado, com co(variância) aditivo-materna fixada como zero. $\mathrm{O}$ modelo de análise na forma matricial encontra-se a seguir:

$$
\mathrm{Y}=\mathrm{Xb}+\mathrm{Z} 1 \mathrm{a}+\mathrm{Z} 2 \mathrm{~m}+\mathrm{e}
$$

em que: Y é o vetor das observaçóes (peso ao nascer); b é o vetor dos efeitos fixos (sexo, ano de nascimento e composição racial do animal); Z1 é a matriz de incidência dos efeitos genéticos aditivo diretos; a é o vetor de efeitos genéticos aditivo diretos do animal; Z2 é a matriz de incidência dos efeitos genéticos aditivos maternos; $\mathrm{m}$ é o vetor de efeito genético aditivo materno; e é o vetor dos erros aleatórios associados às observaçóes.

Os valores iniciais foram obtidos de análise prévia realizada pelo aplicativo REMLF90, com critério de convergência $10^{-12}$. Em seguida, o arquivo de parâmetros foi submetido ao programa GIBBSF90, que usa metodologia Bayesiana com Amostragem de Gibbs para características contínuas, assumindo distribuiçóes iniciais para efeitos genéticos e residuais com distribuiçôes Normais multivariadas, como descrito a seguir:

$$
\begin{aligned}
& P\left(a \mid \sigma^{2} a\right)-N\left(0, A \sigma^{2} a\right) \\
& P\left(e \mid \sigma^{2} e\right)-N\left(0, I \sigma^{2} e\right), \text { em que: }
\end{aligned}
$$

A é a matriz de parentesco, $\sigma^{2}$ a é a variância genética aditiva, $\sigma^{2}$ e é a variância residual e I é uma matriz identidade de ordem $r$ por $r$.

Foram implementados um milhão de iterações, com descarte inicial de 50.000 amostras e amostragem a cada 100 iteraçóes. As saídas passaram a ser trabalhadas no SAS, para calcular as estimativas de herdabilidade direta $\left(h^{2} d\right)$ e materna $\left(h^{2} \mathrm{~m}\right)$, segundo as fórmulas clássicas. Os arquivos gerados pelo SAS foram submetidos às análises a posteriori dos programas GIBANAL (Van Kaam 1997) e POSTGIBBSF90 (Misztál 2001), incluindo a correlação serial entre as amostras e a obtenção das densidades marginais posteriores para confecção dos gráficos de distribuição posterior das herdabilidades, em Excel for Windows. Foi realizado o teste de convergência da cadeia por meio da plataforma R e metodologia BOA (Bayesian Output Analysis).

Os valores genéticos foram obtidos pelas soluçôes geradas no programa GIBBSF90. A regressão dos Valores Genéticos (VGs) sobre o ano de nascimento foi realizada no Excel for Windows para obtenção da tendência genética do peso ao nascer, entre 1974 e 2009 (período que abrangeu o nascimento dos animais presentes no arquivo de pedigree). Foram obtidas tendências genéticas diretas e maternas para a característica de peso ao nascer. 


\section{RESULTADOS}

O peso ao nascer de bezerros bubalinos apresentou média de 36,6 (Tabela 1) e distribuição desviando-se da normal, conforme os valores da curtose (indicando distribuição leptocúrtica) e da assimetria (indicando distribuição assimétrica à esquerda), apresentados na Tabela 1. Este resultado foi comprovado, também pelo teste de Shapiro-Wilk para normalidade dos resíduos.

O critério de Geweke, de análise de convergência da cadeia, foi igual a $Z=0,702163$, indicando que a cadeia MCMC convergiu, por $Z$ estar entre $-1,96<Z<1,96$. A análise das amostras, por meio do programa GIBANAL sugeriu, com base nas correlaçóes seriais, período adicional de descarte e skip time. Com isso, o número efetivo de amostras a serem trabalhadas diminuiu, para cada uma das estimativas (Tabela 2), bem como foram alteradas as correlaçóes seriais e as estatísticas descritivas das variâncias e herdabilidades, ainda que as amostras restantes fossem mais informativas.

Comparando-se os dois gráficos de distribuiçãa das herdabilidades direta (Figura 1) e materna (Figura 2), observase que a primeira apresentou-se platicúrtica (achatada) e com maior assimetria, tendo uma distribuição bimodal com a primeira moda próxima a 0,10 e a segunda próxima a 0,30 . Tendência semelhante ocorreu na distribuição da herdabilidade materna, apresentando-se trimodal, com picos bem próximos ao valor de 0,15 e outro menos evidente próximo a 0,20 .

A tendência genética direta do peso ao nascer mostrou-se negativa $\left(-0,03 \mathrm{~kg} \mathrm{ano}^{-1}\right)$, porém próxima a zero (Figura 3), ainda que a tendência fenotípica tenha sido positiva $(0,156 \mathrm{~kg}$

Tabela 1. Estatística descritiva do peso ao nascer de búfalos do Estado do Pará, Brasil.

\begin{tabular}{cc}
\hline & Estatística \\
\hline $\mathrm{N}$ & 566 \\
\hline Média $\pm \mathrm{DP}(\mathrm{kg})$ & $36,6 \pm 4,7$ \\
\hline Variância $\left(\mathrm{kg}^{2}\right)$ & 21,8 \\
\hline Mediana $(\mathrm{kg})$ & 37 \\
\hline Moda $(\mathrm{kg})$ & 38 \\
\hline Curtose & 0,739 \\
\hline Assimetria & $-0,336$ \\
\hline Mínimo $(\mathrm{kg})$ & 23 \\
\hline Máximo $(\mathrm{kg})$ & 50 \\
\hline
\end{tabular}

$\mathrm{N}$ = número de observações; $\mathrm{DP}=$ desvio-padrão ano $^{-1}$; Figura 4). A tendência materna do peso ao nascer foi próxima a zero, com R-quadrático de valor baixo para ambas as tendências genéticas (Figura 5).

Tabela 2. Correlação serial, Média (Me), moda (Mo), mediana (Med), valor mínimo (Min) e valor máximo (Max) das variâncias genéticas aditiva $(\sigma 2 a)$ e materna $(\sigma 2 \mathrm{~m})$, e das estimativas de herdabilidade direta (h2d) e materna (h2m) para a característica PN, após análise do programa Gibanal.

\begin{tabular}{|c|c|c|}
\hline Estimativas & & Valores \\
\hline \multirow[t]{9}{*}{ Variância genética aditiva $\left(\sigma^{2} a\right)$} & Número de amostras & 475 \\
\hline & Correlação serial & $-0,0591$ \\
\hline & $\mathrm{Me} \pm \mathrm{MCE}^{*}$ & $3,03 \pm 0,0498$ \\
\hline & Mo & 1,71 \\
\hline & Med & 2,65 \\
\hline & Min & 0,03 \\
\hline & Max & 10,40 \\
\hline & Curtose & 0,917 \\
\hline & Assimetria & 0,964 \\
\hline \multirow[t]{8}{*}{ Herdabilidade direta $\left(h^{2} d\right)$} & Número de amostras & 475 \\
\hline & $\mathrm{Me} \pm S D^{* *}$ & $0,17 \pm 0,098$ \\
\hline & Mo & 0,11 \\
\hline & Med & 0,16 \\
\hline & Min & 0,002 \\
\hline & Max & 0,497 \\
\hline & Curtose & 0,193 \\
\hline & Assimetria & 0,729 \\
\hline \multirow[t]{9}{*}{ Variância materna $\left(\sigma^{2} \mathrm{~m}\right)$} & Número de amostras & 1900 \\
\hline & Correlação serial & 0,028 \\
\hline & $\mathrm{Me} \pm \mathrm{MCE}$ & $2,49 \pm 0,018$ \\
\hline & Mo & 2,15 \\
\hline & Med & 2,40 \\
\hline & Min & 0,12 \\
\hline & Max & 6,90 \\
\hline & Curtose & 0,332 \\
\hline & Assimetria & 0,509 \\
\hline \multirow[t]{8}{*}{ Herdabilidade materna $\left(h^{2} m\right)$} & Número de amostras & 1188 \\
\hline & $\mathrm{Me} \pm \mathrm{SD}$ & $0,14 \pm 0,054$ \\
\hline & Mo & 0,14 \\
\hline & Med & 0,14 \\
\hline & Min & 0,01 \\
\hline & Max & 0,36 \\
\hline & Curtose & $-0,017$ \\
\hline & Assimetria & 0,371 \\
\hline
\end{tabular}

* Erro de Monte Carlo; ** Desvio-padrão 


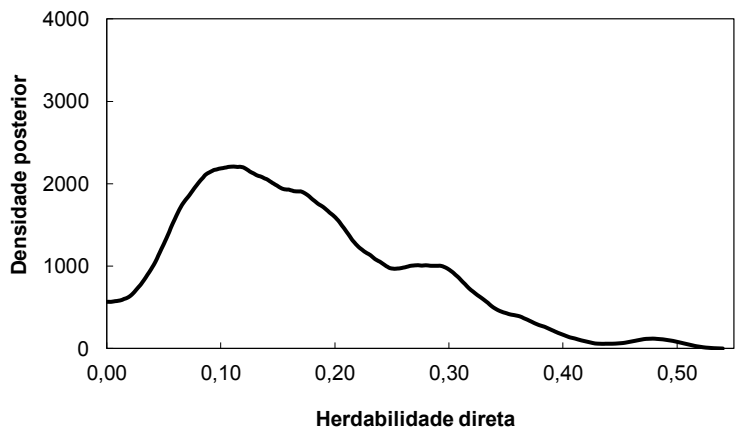

Figura 1. Distribuição marginal posterior da herdabilidade direta do peso ao nascer em bubalinos do Estado do Pará, Brasil

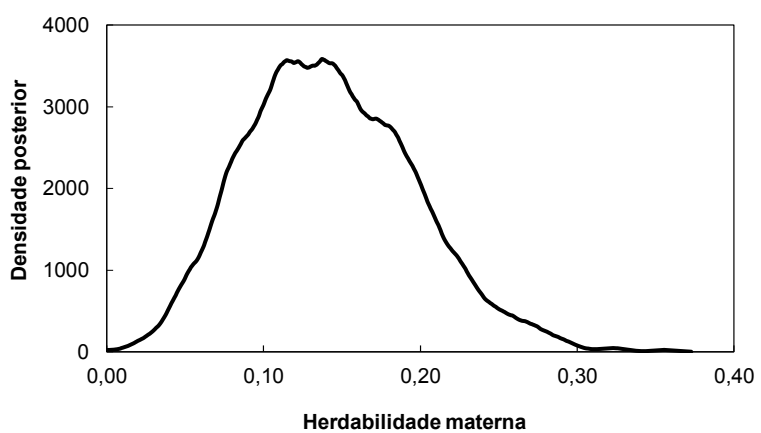

Figura 2. Distribuição marginal posterior da herdabilidade materna do peso ao nascer em bubalinos do Estado do Pará, Brasil

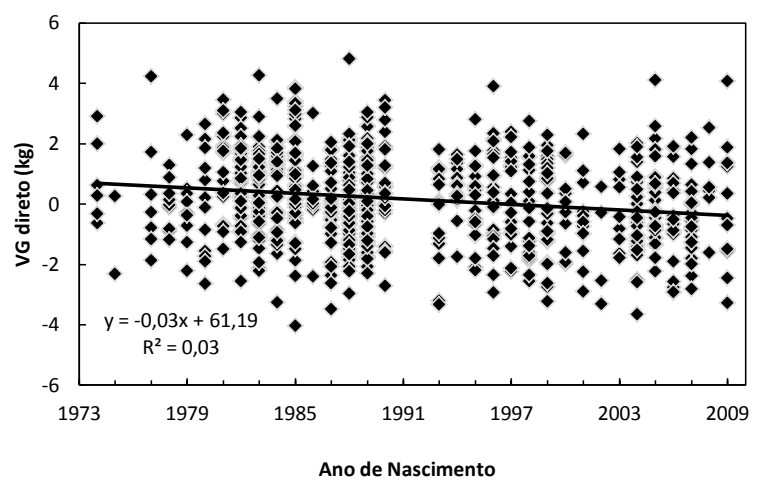

Figura 3. Tendência genética direta do peso ao nascer em bubalinos do Estado do Pará, Brasil. VG direto - Valor Genético direto

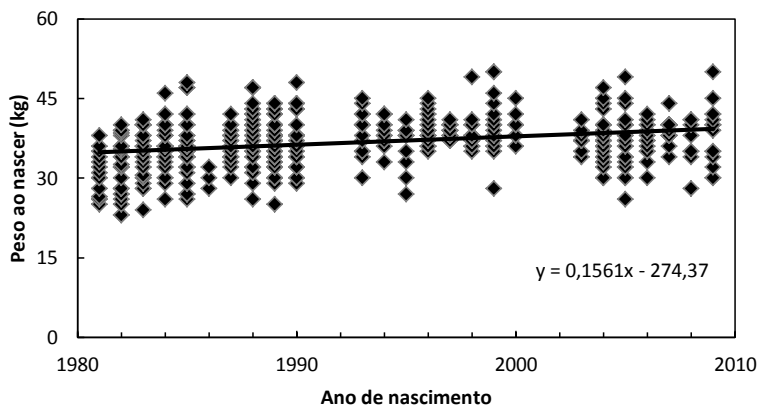

Figura 4. Tendência fenotípica do peso ao nascer em bubalinos do Estado do Pará, Brasil.

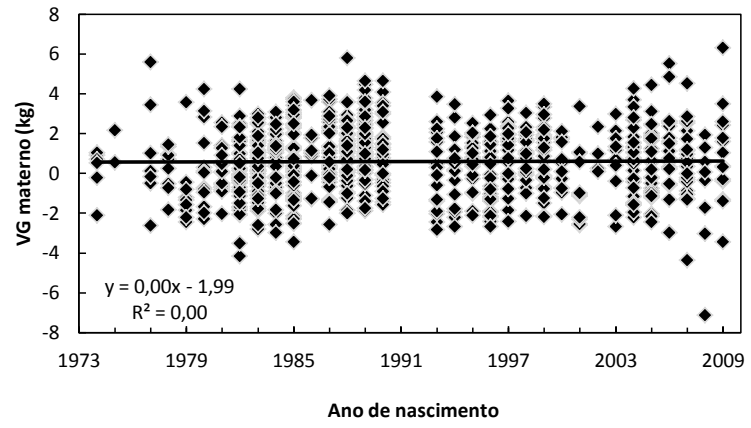

Figura 5. Tendência genética materna do peso ao nascer em bubalinos do Estado do Pará, Brasil. VG materno - Valor Genético materno

\section{DISCUSSÃO}

Pesos ao nascer inferiores (de 27,8 a 34,2 kg; Das et al. 2004; Pereira et al. 2008; Thiruvenkadan et al. 2009) ou superiores (de 37,6 a $42 \mathrm{~kg}$; Jorge et al. 2005; Malhado et al. 2008, Silveira 2012) têm sido relatados e demonstram a variação fenotípica existente na espécie, ainda que estes valores sejam influenciados pelo grupo genético (Cassiano et al. 2003), pelo sexo do bezerro (Jorge et al. 2005; Lopes et al. 2008) e por diversos fatores ambientais e qualidade da pesagem, segundo Malhado et al. (2008). No Estado do Pará, a maior influência é de animais da raça Mediterrâneo e seus mestiços, o maior direcionamento do sistema produtivo para a produção de carne e o clima quente e úmido, que proporciona vigoroso crescimento de pastagens, poderiam explicar as diferenças em algumas médias encontradas para animais da raça Murrah, de outros Estados ou países.

Diferenças quanto aos valores de estimativas de herdabilidade podem ser explicados pela populaçáo estudada, metodologia empregada e modelo de análise. Lopes et al. (2008) encontraram valor de $0,60 \pm 0,12$, por meio do programa DFREML, o que pode ser considerado elevado se comparado aos valores encontrados na literatura para esta característica no site da Association for the Advancement of Animal Breeding and Genetics (AAABG 2010), em sua maioria obtidos por análise de meios-irmáos paternos.

Com aplicação do programa MTDFREML (metodologia de máxima verossimilhança restrita), Cassiano et al. (2004) obtiveram estimativa de 0,62 para a raça Murrah, 0,28 para Jafarabadi e 0,39 para Carabao, enquanto que o valor estimado para a raça Mediterrâneo $(0,16)$ foi próximo àquele de 0,14 encontrado por Mahdy et al. (1999), em búfalos egípcios, indicando que a maior parte da variação seria consequência de fatores ambientais. Já Silveira (2012) utilizando o programa MTGSAM, obteve estimativa de 0,30 para a raça Mediterrâneo, que de acordo com o autor, esse valor indica bons ganhos na seleçáo direta desta característica. Neste estudo, a mediana da herdabilidade direta do peso ao nascer 
foi igual a 0,16 (Tabela 2), valor superior ao encontrado por Thiruvenkadan et al. (2009) na raça Murrah $(0,12)$, mas semelhante ao observado em animais Mediterrâneo por Cassiano et al. (2004) e em búfalos egípcios por Mahdy et al. (1999). Esse valor indica grande influência de fatores de ambiente sobre a característica, no entanto assinala a existência de variabilidade genética que pode ser trabalhada em programas de seleção e melhoramento da espécie.

Quanto à herdabilidade materna, Scarpati e Lôbo (1999) afirmaram que o peso ao nascer seria uma característica pouco influenciada pelo fenótipo materno. Neste estudo, porém, os valores de moda e mediana foram iguais a 0,14 , superiores àqueles obtidos por Cassiano et al. (2004) para a raça Carabao $(0,11)$ e Murrah $(0,04)$ e por Thiruvenkadan et al. (2009) para Murrah $(0,11)$. Todavia esses valores foram inferiores quando comparados com as raças Jafarabadi $(0,17)$ e Mediterrâneo $(0,37)$ no estudo de Cassiano et al. (2004) e com a raça Mediterrâneo $(0,31)$ no estudo de Silveira (2012), raças essas caracterizadas por seu maior tamanho corporal. Os valores encontrados para herdabilidade materna foram expressivos e evidencia a influência do ambiente uterino e demais fatores que relacionados à gestação que podem interferir no peso ao nascer dos animais, além da variação entre indivíduos.

Em raças especializadas para a produção de carne, como a raça Nelore (Ferraz Filho 1996; Holanda et al. 2004) e a raça Canchim (Mello et al. 2002) as tendências genéticas dos efeitos diretos para o peso ao nascer variaram de 0,002 a $0,083 \mathrm{~kg} \mathrm{ano}^{-1}$. Em termos de mudança genética anual, isto representa incrementos abaixo de $0,5 \%$ nas médias da característica e diferenças abaixo de $0,500 \mathrm{~kg}$ para uma geração de seleçáo. Isso indica que a seleção para crescimento adotada no rebanho náo estaria promovendo alteraçóes de grande magnitude no peso ao nascer dos animais. Para os bubalinos paraenses a tendência mostrou-se semelhante, evidenciando a pouca pressão de seleção para peso e ganhos de peso nos rebanhos estudados, já que as correlaçôes genéticas são altas e positivas entre os pesos nas diferentes idades. Em relação à tendência materna, Holanda et al. (2004) obtiveram valores na ordem de $-0,80 \mathrm{~g} \mathrm{ano}^{-1}$, na raça Nelore, a qual não possui seleção para aumento da produção de leite, como ocorre nas fêmeas bubalinas.

O foco em padrão racial, existente entre os criadores que participam de exposiçóes, isto é, a maior importância dada aos caracteres qualitativos dissociados das características produtivas provavelmente ainda é um grande entrave ao processo seletivo e ao completo entendimento das açóes relacionadas ao melhoramento genético dos rebanhos. Por outro lado, pela variabilidade genética existente há possibilidade de se trabalhar os acasalamentos para que, futuramente, não ocorram partos distócicos em grandes proporçóes, como é o caso da raça Charolês (Fernandes et al.
2002) ou mesmo em animais mestiços (Borges et al. 2006), sem que no entanto, cause prejuízos com baixo desempenho e vigor dos bezerros. O estudo do peso ao nascer e suas relaçóes com outras características de crescimento permite aos programas de seleção o monitoramento deste fenótipo, tornando sua aplicabilidade importante nas diferentes espécies domésticas.

\section{CONCLUSÕES}

Existe variabilidade genética nos rebanhos estudados que pode ser trabalhada em um programa de seleção e melhoramento genético, dadas às estimativas de herdabilidade direta e materna. Pouco foi feito em relação à seleção para peso nos búfalos paraenses às diferentes idades.

\section{AGRADECIMENTOS}

Aos criadores parceiros do Programa de Melhoramento Genético de Búfalos (APCB/Embrapa/UFRA), à Coordenação de Aperfeiçoamento de Pessoal de Nível Superior (CAPES) pela Bolsa da aluna Juliana, ao Conselho Nacional de Desenvolvimento Científico e Tecnológico (CNPq), à Fundação Amazônia Paraense de Amparo à Pesquisa (FAPESPA) e à Empresa Brasileira de Pesquisa Agropecuária (EMBRAPA) pelo financiamento do projeto.

\section{BIBLIOGRAFIA CITADA}

AAABG - Association for the Advancement of Animal Breeding and Genetics, 2010. Genetic parameters. (http://www.gparm. csiro.au/traitgroups.html?species=7). Acesso em 20/01/2010.

Borges, M.C.B.; Costa, J.N.; Ferreira, M.M.; Menezes, R.V.; Chalhoub, M. 2006. Caracterização das distocias atendidas no período de 1985 a 2003 na Clínica de Bovinos da Escola de Medicina Veterinária da Universidade Federal da Bahia. Revista Brasileira de Saúde e Produção Animal, 7: 87-93.

Cassiano, L.A.P.; Mariante, A.S.; McManus, C.; Marques, J.R.F.; Costa, N.A. 2003. Caracterização fenotípica de raças bubalinas nacionais e do tipo Baio. Pesquisa Agropecuária Brasileira, 38: 1337-1342.

Cassiano, L.A.P.; Mariante, A.S.; McManus, C.; Marques, J.R.F.; Costa, N.A. 2004. Parâmetros genéticos das características produtivas e reprodutivas de búfalos na Amazônia brasileira. Pesquisa Agropecuária Brasileira, 39: 451-457.

Das, A.; Das, D.; Goswami, R.N.; Bhuyan, D. 2004. Growth performance of swamp buffaloes of Assam from birth to 12 months of age. Buffalo Bulletin, 23: 84-89.

Fernandes, H.D.; Ferreira, G.B.B.; Rorato, P.R.N. 2002. Tendências e parâmetros genéticos para características pré-desmama em bovinos da raça Charolês criados no Rio Grande do Sul. Revista Brasileira de Zootecnia, 31: 321-330.

Ferraz Filho, P.B. 1996. Análise e tendência genética de pesos em bovinos da raça Nelore Mocha no Brasil. Dissertação de Mestrado, Universidade Estadual Paulista, Jaboticabal, São Paulo. 163p. 
Holanda, M.C.R.; Barbosa, S.B.P.; Ribeiro, A.C.; Santoro, K.R. 2004. Tendências genéticas para crescimento em bovinos Nelore em Pernambuco, Brasil. Archivos de Zootecnia, 53: 185-194.

Jorge, A.M.; Andrighetto, C.; Castro, V.S. 2005. Desenvolvimento ponderal de bubalinos da raça Murrah criados em pastagem de Brachiaria brizantha no Centro-Oeste do Estado de São Paulo, Brasil. Ciência Rural, 35: 417-421.

Lopes, C.R.A.; Barbosa, S.B.P.; Pereira, R.G.A.; Santoro, K.R.; Lira, A.V. 2008. Eficiência reprodutiva e influência de fatores de meio e de herança sobre a variação no peso ao nascer de bubalinos no estado de Rondônia. Revista Brasileira de Zootecnia, 37: 1595-1600.

Mahdy, A.E.; El-Shafie, O.M.; Ayyat, M.S. 1999. Genetic study and sire values for some economic traits in Egyptian buffaloes. Alexandria Journal of Agricultural Research, 44: 15-35.

Malhado, C.H.M.; Ramos, A.A.; Carneiro, P.L.S.; Azevêdo, D.M.M.R.; Martins Filho, R. 2008. Distribuição univariada e bivariada das características de crescimento de bubalinos de corte no Brasil. Revista Científica de Produção Animal, 10: 69-80.

Mello, S.P.; Alencar, M.M.; Silva, L.O.C.; Barbosa, R.T.; Barbosa, P.F. 2002. Estimativas de (co) variâncias e tendências genéticas para pesos em um rebanho Canchim. Revista Brasileira de Zootecnia, 31: 1707-1714.

Misztál, I. 2001. Computer programs. (http://nce.ads.uga. edu/ ignacy/programs.html). Acesso em 20/04/2010.
Nogueira, J.R.; Cardoso, V.L.; Mattos, J.C.A.; Lima, M.L.P.; Camargo, D.F.V. 1997. Sistema de produçáo de bubalinos para carne em pastagens de capim colonião na região Noroeste do Estado de São Paulo. Boletim de Indústria Animal, 54: 25-32.

Pereira, J.C.C. 2008. Melhoramento genético aplicado à produção animal. 5.ed. Belo Horizonte, FEPMVZ Editora, 618p.

Pereira, R.G.A.; Barbosa, S.B.P.; Lopes, C.R.A.; Santoro, K.R.; Townsend, C.R.; Magalhães, J.A.; Silva Netto, F.G.; Costa, N.L. 2008. Peso ao nascer em bubalinos no Estado de Rondônia. Porto Velho. Embrapa Rondônia, 54: 12 p.

SAS 2002. Statistical Analysis System. SAS Users Guide: Statistics, Version 9.1.

Scarpati, M.T.V.; Lôbo, R.B. 1999. Modelos animais alternativos para estimaçáo de componentes de (co) variância e de parâmetros genéticos e fenotípicos do peso ao nascer na raça Nelore. Revista Brasileira de Zootecnia, 28: 512-518.

Silveira, E.S. 2012. Parâmetros genéticos para características de crescimento em bubalinos da raça Mediterrânea estimados por Inferência Bayesiana. Dissertação de Mestrado, Universidade Estadual do Sudoeste da Bahia, Itapetinga, Bahia. 55p.

Thiruvenkadan, A.K.; Panneerselvam, S.; Rajendran, R. 2009. Nongenetic and genetic factors influencing growth performance in Murrah Buffalos. South African Journal of Animal Science, 39: 102-106.

Van Kaam, J.B.C.H.M. 1997. GIBANAL: Analysing program for Markov Chain Mont Carlo sequences version 2.3.

Recebido em 05/07/2013

Aceito em 23/01/2014 